Research Article

Piotr Lipinski* and Marcin Leplawy

\title{
WiFi Electromagnetic Field Modelling for Indoor Localization
}

https://doi.org/10.1515/phys-2019-0039

Received Jan 28, 2019; accepted Feb 12, 2019

\begin{abstract}
The aim of this paper is to develop simplified model for WiFi electromagnetic field propagation. The model can be used in preliminary estimation of WiFi transmitter localization for the purpose of WiFi-based indoor localization. It can be particularly useful when designing structural networks. We have developed a simplified linear model of WiFi electromagnetic field modelling and compared it with the most commonly used, more sophisticated models and with measurement results which were carried out in the laboratory. As demonstrated by the results of measurements conducted using various hardware, the accuracy of this simplified model introduced is similar to the commonly used models, but the number of parameters is lower. Therefore, our model easier to implement in real life conditions. The model presented in this paper enables WiFi electromagnetic field modelling when the exact values of propagation parameters and transmitter characteristics is unknown. This is usually the case at the early stage of structural network design, when exact parameters of building construction materials are not known. As the model is very simple, it does not require much effort to deploy, while its accuracy is sufficient for preliminary WiFi transmitter localization. Simplified models of WiFi electromagnetic field propagation are known, but no comparative research combined with measurements has been done in this field. This paper provides a comparison of different electromagnetic field models which can be applied to WiFi electromagnetic field propagation together with measurement results.
\end{abstract}

Keywords: WiFi localization modelling, fingerprinting, indoor localization modelling

PACS: 41.20.Jb, 07.05.Tp, 41.20.-q

\footnotetext{
^Corresponding Author: Piotr Lipinski: Institute of Information Technology, Lodz University of Technology, Lodz, Poland; Email: piotr.lipinski@p.lodz.pl

Marcin Leplawy: Institute of Information Technology, Lodz University of Technology, Lodz, Poland; Email: marcin.leplawy@dokt.p.lodz.pl
}

\section{Introduction}

WiFi is a technology for wireless local area networking which bases on the IEEE 802.11 standards. It most commonly uses the $2.4 \mathrm{GHz}, 3.6 \mathrm{GHz}, 5.8 \mathrm{GHz}, 60 \mathrm{GHz}$ and $900 \mathrm{GHz}$ radio frequencies, see (IEEE 802.11, Wireless LAN Medium Access Control (MAC) and Physical Layer (PHY) specifications IEEE, 2007.). Frequency assignments and operational limitations are different worldwide. Australia and Europe allow for two channels beyond the eleven permitted in the United States for the $2.4 \mathrm{GHz}$ band. In Japan an extra three more channels are available. Nevertheless in most countries for IEEE $802.11 \mathrm{~b} / \mathrm{g} / \mathrm{n}$ standards the wave frequency do not exceed $2.4 \mathrm{GHz}-2.5 \mathrm{GHz}$ range. For $802.11 \mathrm{a} / \mathrm{h} / \mathrm{j} / \mathrm{n} / \mathrm{ac} / \mathrm{ax}$, the range $5.15 \mathrm{GHz}-5.725 \mathrm{GHz}$ is used, for $802.11 \mathrm{ad} /$ ay the range $57.24 \mathrm{GHz}-70.2 \mathrm{GHz}$ is used and for 802.11ah $863 \mathrm{GHz}-868 \mathrm{GHz}$ is used in Europe, see (IEEE 802. Annex J modified by amendments $k, y$ and $n, 2007)$. WiFi and other wireless standards are nowadays used in a majority of public buildings, see Peulic et al. (2007) [1]. A WiFi emitter uses an antenna to propagate a wireless electromagnetic signal according to antenna characteristics Jevtić and Ničković (2011) [2]. The strength of this signal can be measured by using a WiFi receiver for indoor localization, see for example Chen (2008) [3], Connolly (2008) [4]. The strength of the signal depends on the following parameters: power of the source, antennas' characteristics, distance from the source of the signal, magnetic permeability, permittivity and refraction according to Kong et al. (2011) [5]. Very accurate modelling of magnetic field is very difficult and time consuming, see Napieralski et al. (2015) [6]. Furthermore, it requires accurate values of the abovementioned parameters for all the materials which are used in the building construction (Motley and Keenan 1990) [7] as well as objects and humans which is usually difficult to achieve, see Siauve et al. (2003) [8], López et al. (2015) [9], Glonek G. et al. [10]. Therefore simplified models are used to make the calculation faster and parameter acquisition easier, see Yu and Sebo (1996) [11] and Todaka et al. (2009) [12]. Here we demonstrate that a linear model for electromagnetic wave propagation and approximate values of magnetic permeability, permittivity

๑ Open Access. () 2019 P. Lipinski and M. Leplawy, published by De Gruyte 
and refraction together with simplified antenna propagation model are sufficient to obtain preliminary WiFi access point localization in the building, when WiFi-based in door localization is considered to be deployed in the building. There are many available electromagnetic field models and software solutions for electromagnetic field simulation (Mora et al. 2006) [13], but the most common model for indoor WiFi attenuation involves the path loss exponent which accounts for all propagation phenomenon. For example consider the one-slope model where the loss term is given by Hashemi (1993) [14]:

$$
L=L_{0}+10 \cdot n \cdot \log (d),
$$

where:

$L_{0}$ - unit loss,

$d$-distance,

$n$ - power decay index, which depends on material.

This model takes into account the increasing path loss owe to the penetration of obstacles such as walls and floors by varying the power decay index with the distance.

More sophisticated approaches take into account the decreasing penetration loss of walls of the same category as the number of traversed walls increase. The walls that have to be considered are determined by the Obstructed Line-Of Sight path. The parameters of the model can be found in Lott and Forkel (2001) [15]. In this model the loss term is given as follows:

$$
L=L_{0}+10 \cdot n \cdot \log (d)+\sum_{i=1}^{l} \sum_{k=1}^{K_{w i}} L_{w i k},
$$

where: $L_{0}$ - unit loss,

$d$-distance,

$n$ - power decay index, which depends on material

$L_{w i k}-$ attenuation due to wall type i and k-th traversed wall,

$I$ - number of wall types,

$K_{w i}-$ number of traversed walls of category $i$.

\section{Simplified model}

Here we introduce a simplified 2D linear model for indoor WiFi electromagnetic signal distribution modelling. The motivation for introducing such a simple model are measurement results which demonstrate, that when the model is used for RSSI localization it provides satisfactory results. The model takes the following form:

$$
L_{p L}=L_{0 L}+L_{1} \cdot d+\sum_{i=1}^{l} \sum_{k=1}^{K_{w i}} L_{w i k},
$$

where: $L_{0 L}-$ unit loss in linear model, $d$-distance,

$L_{L w i k}$ - attenuation due to wall type $i$ and $k$-th traversed wall in linear model,

$I$ - number of wall types,

$K_{w i}$ - number of traversed walls of category $i$.

The attenuation $L_{S w i k}$ for most common building materials are given in Table 1.

Table 1: Average localization error results for classical and simplified models for different testbeds

\begin{tabular}{ccc}
\hline No & Wall material & $\mathrm{dB} / \mathrm{cm}$ \\
\hline 1 & Wood & 0,2 \\
2 & Gypsum wall & 0,2 \\
3 & Glass & 10 \\
4 & Double glazed window & 6 \\
5 & Concrete & 0,35 \\
\hline
\end{tabular}

Comparison between this simplified model and the classical model in 1D for desired attenuation and distance range is depicted in Figure 1. It is clearly visible that simplified model and classical model differs very little within the considered attenuation and distance range. Therefore, the simplified model can effectively simulate WiFi electromagnetic field propagation. In order to verify if the simplified model can be practically used we have carried out experiments comparing the model with measurement results.

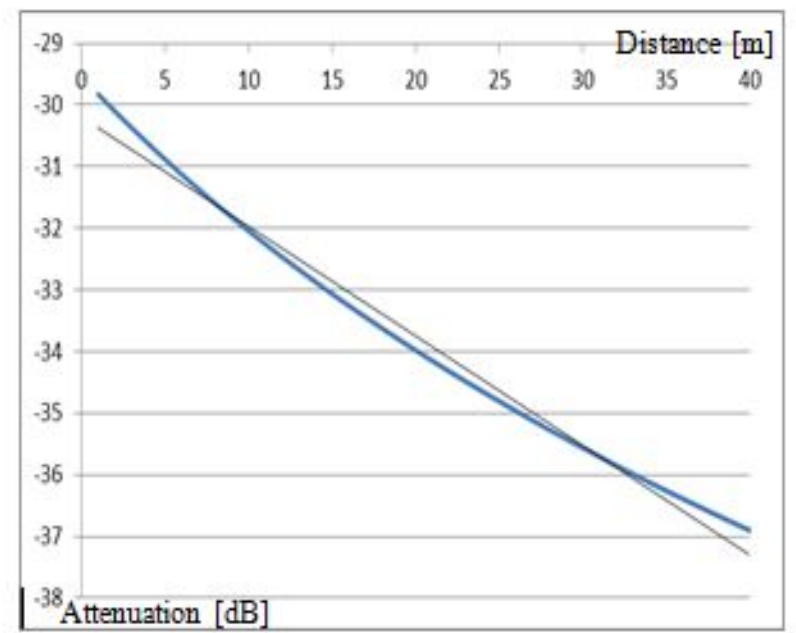

Figure 1: Classical vs simplified model of WiFi electromagnetic field RSSI. 


\section{Experimental verification of the model}

The experiments were carried out in one of the laboratories situated at Lodz University of Technology. In these experiments several different receivers and transmitters were used as well as 5 different wall materials. The simplified model of WiFi signal propagation was compared with the measurement results in open space as well as when passing through: wooden, gypsum, glass and concrete wall. The experiment was organized as follows: For each device pair (transmitter-receiver) two WiFi access points were located in two corners of the measurement area and one was located in the middle of the side of the measurement area. Next, the receiver (raspberry computer or a mobile phone) was placed on a robot. The robot followed the line on the floor using an optical sensor and captured WiFi RSSI value in measurement points marked on the floor, see Figure 2. Each measurement was repeated 10 times for each transmitter-receiver pair. As a result for each transmitterreceiver pair we were collecting 36 measurement values (one per each measurement point). As the measurement was repeated 10 times, we were collecting 360 measurements total for each transmitter-receiver pair. Thanks to the use of the robot, the measurements were carried out under the same conditions. In this experiment we have tested several models of WiFi transmitters together with several WiFi receivers (smartphones and Rasberry Pi computers). It should be noted that each transmitter has different WiFi electromagnetic field characteristics, which is influenced by its design, antenna's shape, number of antennas etc. WiFi RSSI receivers are also of concern as each has different design, sensitivity and antenna characteristics. If one tries to create a very accurate model, it requires taking into consideration all the above mentioned factors which are modelled by many parameters. Hence it is very difficult to model the electromagnetic field propagation for the purpose of WiFi localization. Therefore, it is important to develop simplified models, which are not very accurate, but can be used in practise when all precise parameters are difficult to obtain. Unfortunately, simplified models may lead to large measurement errors and inaccuracy. This is why we tested different hardware combinations in order to demonstrate the usefulness and accuracy of the simplified model. In case of the WiFi transmitter the antenna type strongly influences electromagnetic field propagation, therefore we have tested the hardware with four different antenna solutions:

1. Internal antenna;
2. One external antenna;

3. Two external antennas;

4. Three external antennas.

The following devices were used as WiFi transmitters:

1. Zyxel NWA5160N (square-shaped internal antenna)

2. Zyxel NWA5121-NI (rounded-shape internal antenna)

3. Zyxel NWA5123 (rounded-shape internal antenna with double transmitter

4. Asmax WiFi BR-604G (one external antenna)

5. TP-LINK TL-MR3220 (two external antennas)

6. Cisco AP541N-A-K9 (three external antennas)

For each experiment two WiFi transmitters of the same model were located in the corners of test track and one was located in the middle of the opposite side of the track (see Figure 2).

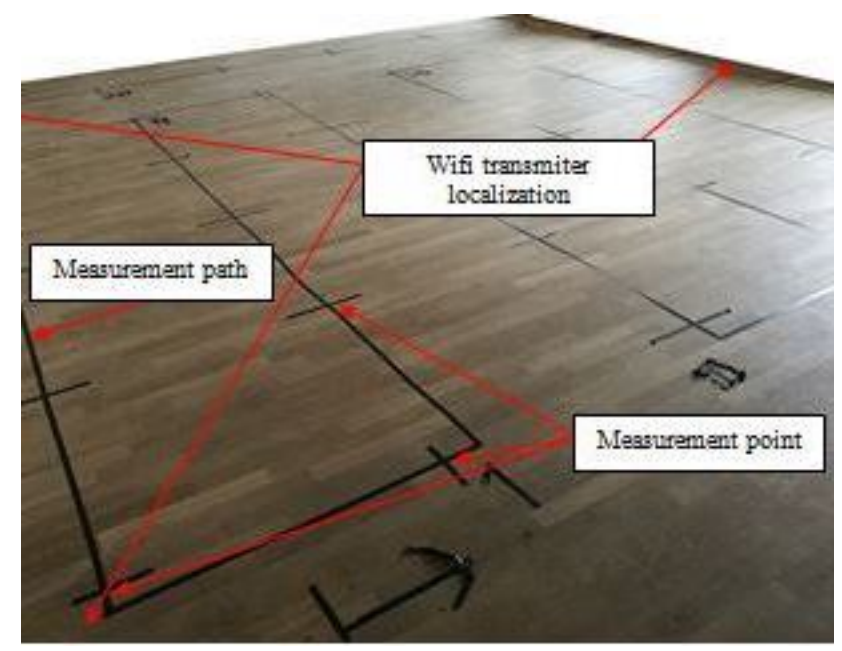

Figure 2: Testbed for measuring electromagnetic field propagation as a function of distance for WiFi localization.

When considering WiFi-based indoor localization the electromagnetic field value is usually measured using a mobile phone or single-board computer. As a result not only the transmitter influences the measurement, but also the receiver, which has the parameters which are not always available to the user. Therefore we have used a variety of mobile devices in our experiments:

1. TP Link TL-wr740n

2. LG g3s

3. $L G g 4 \mathrm{~S}$

4. Samsung Galaxy grand prime sm-g531 h/ds

5. LG g3 LS990

6. Huawei P8 Lite 2017

7. Meizu M3S 

8. Xperia XA
9. Lenovo $\mathrm{P} 70$
10. Fly era3
11. LG g3
12. Xiaomi Redmi 4
13. Nexus $5 \mathrm{X}$
14. Samsung Galaxy J3
15. Kruger\&Matz Flow
16. Sony Xperia $G$
17. Samsung J3

We tested all combinations of transmitter-receiver pairs from the above lists. All the experiments were carried out in open space, as depicted in Figure 2, as well as with the wall made of the materials listed in Table 1 . The wall was located between measurement points, as depicted in Figure 3.

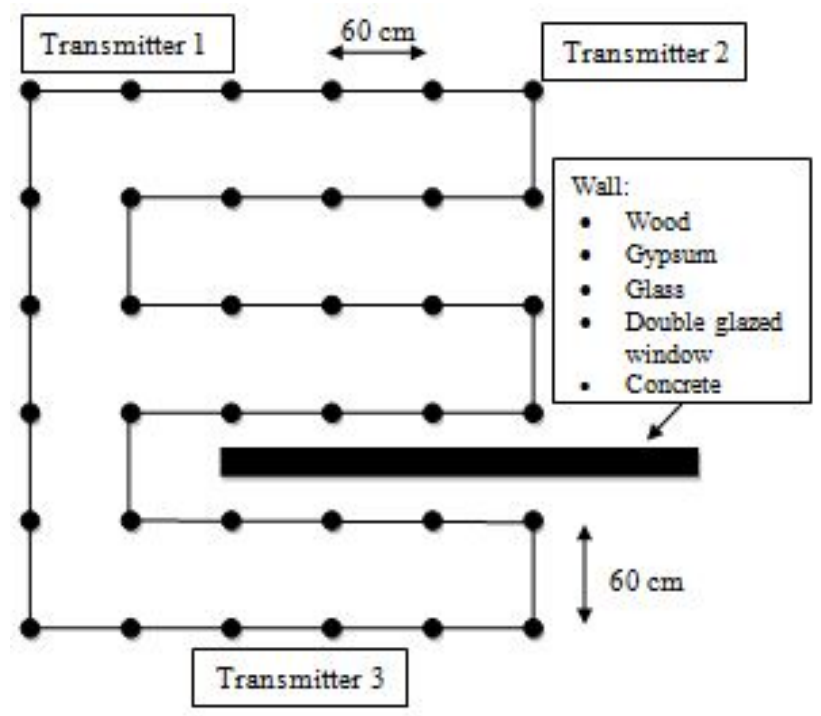

Figure 3: Testbed scheme for measuring electromagnetic field propagation as a function of distance for Wifi localization.

\section{Experimental results}

We have conducted a large number of experiments. Altogether experiments were carried out for $6 \mathrm{WiFi}$ transmitter models, $17 \mathrm{WiFi}$ receivers, and 7 tracks (including different walls and open space). For each measurement point measurements were repeated 10 times, which gives a total of over 257000 measurements. Therefore here we demonstrate only selected results and averaged summary. Experimental results were compared to the results of simulation using the classical model (2) and simplified linear model (3). The results shown in Figures 4 - 6 correspond to the measurement scheme from Figure 3. The Figures 4 - 6 show the electromagnetic fileld distribution for the TP-LINK TLMR3220 transmitters and TP Link TL-wr740n receiver. Figures 4 - 6 show the results for transmitter 1 from Figure 3.

It is clearly noticeable that the results differ for different transmitter and wall localization in Figures 4 - 6. Nevertheless, when one analyses larger number of measurement results it is difficult to infer which model is better in general. Therefore we have computed average errors for different transmitter-receiver pairs, as we want to create a general model. These average results are for: open space, wooden wall, gypsum wall, glass, double glazed window and concrete wall, as shown in Table 2 . The results from Table 2 demonstrate that the simplified model outperforms classical model in terms of average error for all considered testbeds.

Table 2: Average results of mean error for classical and simplified models for different testbeds

\begin{tabular}{cccc}
\hline No & Testbed & $\begin{array}{c}\text { Classical } \\
\text { model mean } \\
\text { error [dB] }\end{array}$ & $\begin{array}{c}\text { Simplified } \\
\text { model mean } \\
\text { error [dB] }\end{array}$ \\
\hline 1 & Open space & 2,76 & 2,34 \\
2 & Wood & 2,61 & 2,59 \\
3 & Gypsum wall & 2,78 & 2,59 \\
4 & Glass & 2,69 & 2,51 \\
5 & Double glazed & 2,66 & 2,49 \\
6 & window & & \\
6 & Concrete & 2,55 & 2,55 \\
\hline
\end{tabular}

The next step of our experiment was to verify if a simplified model can be used for modelling WiFi electromagnetic field propagation for WiFi-based localization. In order to do this we have computed localization of the robot basing on: average measurement results, classical model and simplified model for each measurement point. Next, we have compared the localization result with ground truth, that is the actual localization of the robot. The average localization error results are presented in Table 3. It can be clearly noticed that the simplified model performs slightly better in terms of WiFi-based localization. 


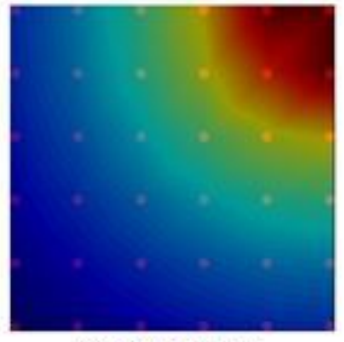

Classical model

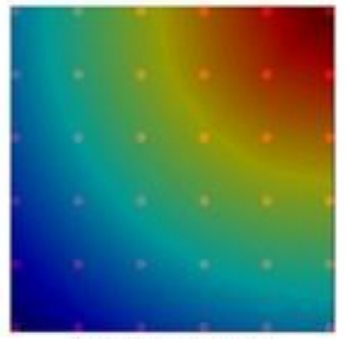

Simplified model

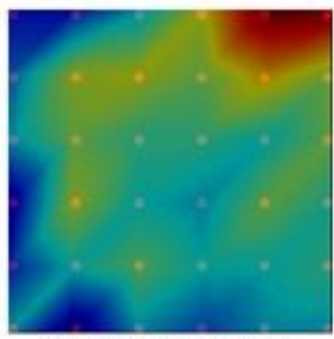

Measurement results

Figure 4: WiFi electromagnetic field propagation for transmitter 2, open space.

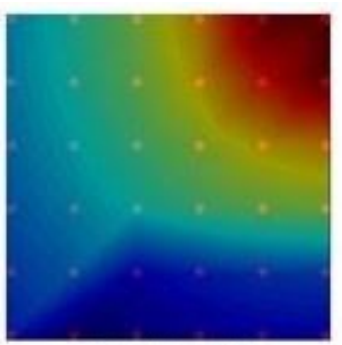

Classical model

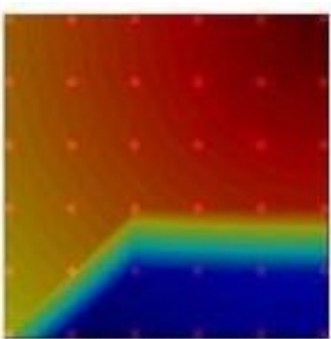

Simplified model

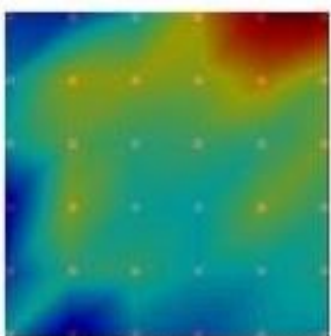

Measurement results

Figure 5: WiFi electromagnetic field propagation for transmitter 2, wooden wall.

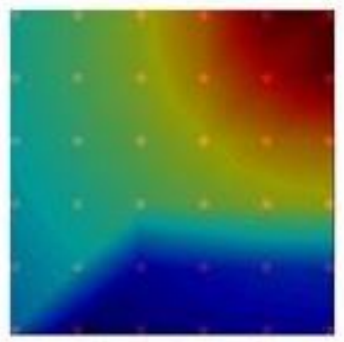

Classical model

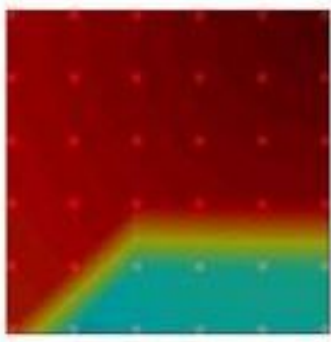

Simplified model

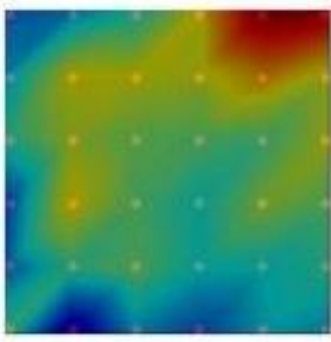

Measurement results

Figure 6: WiFi electromagnetic field propagation for transmitter 2, concrete wall.

Table 3: Average localization error results for classical and simplified models for different testbeds

\begin{tabular}{ccccc}
\hline No & Testbed & $\begin{array}{c}\text { Classical model based } \\
\text { localization error }[\mathrm{m}]\end{array}$ & $\begin{array}{c}\text { Simplified model based } \\
\text { localization error [m] }\end{array}$ & $\begin{array}{c}\text { Measurement-based } \\
\text { localization error [m] }\end{array}$ \\
\hline 1 & Open space & 2,53 & 2,43 & 2,03 \\
2 & Wood & 3,05 & 2,95 & 2,55 \\
3 & Gypsum wall & 2,89 & 2,79 & 2,39 \\
4 & Glass & 3,38 & 3,28 & 2,88 \\
5 & Double glass window & 2,95 & 2,85 & 2,45 \\
6 & concrete & 3,41 & 3,31 & 2,91 \\
\hline
\end{tabular}




\section{Conclusions}

In this paper a novel, simplified linear model for electromagnetic field propagation for the purpose of WiFi localization has been introduced. It considers linear penetration loss of obstacles in the decibel scale. The proposed model is appropriate for many real life applications, such as preliminary WiFi placement and WiFi localization, as it is easy to use and accurate enough even for complex buildings. The main contribution of this work is the study of multiple hardware experiments which have proved that the simplified model can be used despite the heterogeneity of the hardware which is used in WiFi networks. Nevertheless, it should be clear that the experiments presented in this article are not complete when it comes to generalization of the model but they give decent information about possibility of using a simplified linear model for WiFi electromagnetic field propagation for the purpose of WiFi-based localization.

\section{References}

[1] Peulic A., Pesovic U., Cucej Z., ZigBee wireless network performance analysis and electromagnetic fields modelling, 14th International Workshop on Systems, Signals and Image Processing and 6th EURASIP, 2007, 349-351.

[2] Jevtić R. B., Ničković J. T., Measuring and simulation electromagnetic field results of wireless router AT-WA1104G, 19thTelecommunications Forum Proceedings of Papers, 2011, 293-296.

[3] Chen Y., Chandrasekaran G., Elnahrawy E., Francisco J.A., Kleisouris K., Li X., et al., GRAIL: a general purpose localization system, Sensor Review, 28, 2, 2008.

[4] Connolly C., Developments in localisation sensor systems, Sensor Review, 2008, 2, 28.
[5] Kong S., Hwang Ch., Kim K, Kim H., Park L, et al., Analytical model for predicting the electromagnetic fields intensity in wireless power transfer systems, IEEE Electrical Design of Advanced Packaging and Systems Symposium, 2011, 1-4.

[6] Napieralski P., Kowalczyk M., Detection of vertical disparity in three-dimensional visualizations, Open Physics, 2017, 15 (1).

[7] Motley A., Keenan J., Radio Coverage in Buildings British Telecom Tech., 1990, 8 (1), 19-24.

[8] Siauve N., Scorretti R., Burais N., Nicolas L., Nicolas A., Electromagnetic fields and human body: a new challenge for the electromagnetic field computation, COMPEL - The international journal for computation and mathematics in electrical and electronic engineering, 2003, 3, 22.

[9] López A.N., Gonzalez-Rubio J., Villalba Montoya J.M., Garde E.A., Using multiple exposimeters to evaluate the influence of the body when measuring personal exposition to radio frequency electromagnetic fields, COMPEL - The international journal for computation and mathematics in electrical and electronic engineering, 2015, 4, 34

[10] Glonek G., Wojciechowski A., Hybrid Orientation Based Human Limbs Motion Tracking, Sensors, 2017, 17(12), 2857.

[11] Yu Q., Sebo S.A., Simplified Magnetic Field Modeling and Calculation of Large Air-Core Reactor Coils, IEEE Transactions On Magnetics, 1996, 5, 32.

[12] Todaka T., Nakanoue K., Enokizono M., Magnetic field analysis under complex approximation taking account of two-dimensional magnetic properties. COMPEL - The international journal for computation and mathematics in electrical and electronic engineering, 2009, 1, 28.

[13] Mora J., Otín R., Dadvand P., Escolano E., Pasenau M.A., Oñate E., Open tools for electromagnetic simulation programs, COMPEL - The international journal for computation and mathematics in electrical and electronic engineering, 2006, 3, 25.

[14] Hashemi H., The Indoor Radio Propagation Channel, Proc. of the IEEE, 81 (7), 1993, 943-968.

[15] Lott M., Forkel I., A multi-wall-and-floor model for indoor radio propagation IEEE VTS 53rd Vehicular Technology Conference Spring 2001. Proceedings, 2001, 464-468. 